Original Article

\title{
Intermittent administration of parathyroid hormone improves bone quality and quantity around implants in rat tibiae
}

\author{
Yusuke Uchida, Shinichiro Kuroshima*, Yusuke Uto, Riho Kanai, Maaya Inoue, \\ Masayoshi Suzue, Takashi Sawase \\ Department of Applied Prosthodontics, Graduate School of Biomedical Sciences, Nagasaki University, Nagasaki, 852-8588, Japan
}

\section{A R T I C L E I N F O}

\section{Article history:}

Received 22 February 2020

Accepted 28 February 2020

Available online 6 April 2020

\section{Keywords:}

Bone quality

Bone cells

Parathyroid hormone

Implant

\begin{abstract}
A B S T R A C T
Objectives: Intermittent injection of parathyroid hormone $(\mathrm{PTH})$ is used to treat osteoporosis. The concept of bone quality was updated 20 years ago; however, these updates have not been adopted in implant dentistry. This study aimed to investigate the effects of intermittent administration of PTH on bone quality around implants in rat tibiae.

Methods: Grade IV-titanium-threaded implants that were $3.5 \mathrm{~mm}$ long and $2.0 \mathrm{~mm}$ wide were placed in a randomly selected side of the proximal tibiae of 12-week-old female Wistar rats. Three weeks after implant placement, the rats were randomly divided into PTH-administration and saline-injection groups (PTH and VC, respectively; $\mathrm{n}=7$ per group). Micro-computed tomographical, histomorphometric, and immunohistochemical analyses were performed to evaluate bone quality and quantity surrounding the implants.

Results: PTH significantly increased bone volume and bone mineral density in bones not associated with the implants as compared to these values in the VC group. PTH significantly increased bone area and the amount of collagen within the total inside areas of all implant threads compared to that observed in VC. Moreover, PTH significantly increased the number of osteoblasts, osteocytes, and osteoclasts in the total inside and/or outside areas of all implant threads and altered the ratio of type I and III collagen to total collagen fibers.

Conclusions: Within the limitations of this study, intermittent administration of PTH improved both bone quantity and bone quality based on the types and numbers of bone cells and the types of collagen fibers surrounding implants placed into rat tibiae.
\end{abstract}

(c) 2020 Japanese Association for Oral Biology. Published by Elsevier B.V. All rights reserved.

\section{Introduction}

Implant therapy is a major treatment option for missing teeth. Osseointegration surrounding dental implants is an important factor for determining the long-term clinical outcome of an implant [1]. Additionally, both bone volume and bone mineral density (BMD) in the jawbone are contributing factors [2,3]. In implant dentistry, BMD plays an important role in determining bone strength relative to bone volume, and it is believed to be synonymous with bone quality [4]. Bone quality is conventionally evaluated by bone toughness during the preparation of implant beds by drilling alveolar bone. However, in 2000, the National Institutes of

\footnotetext{
* Corresponding author. Department of Applied Prosthodontics, Graduate Schoo of Biomedical Sciences, Nagasaki University, Nagasaki, 1-7-1, Sakamoto, Nagasaki, 852-8588, Japan.

E-mail address: kuroshima@nagasaki-u.ac.jp (S. Kuroshima).
}

Health (NIH) proposed the new evaluation parameter "bone quality" [5]. According to this proposal, bone quality is completely independent of BMD. Bone quality is defined as "the sum of all characteristics of bone that influence the bone's resistance to fracture" [6] and refers to bone structures, bone turnover, mineralization, and damage accumulation [5]. We previously demonstrated that these components of bone quality are strongly correlated with bone cells, collagen fibers, and biological apatite under mechanical-loaded conditions [7-10].

Parathyroid hormone (PTH), which is produced by the parathyroid grand, controls serum calcium levels through its influences on bone tissue, the kidneys, and the small intestine. Animal and clinical studies clearly reveal that intermittent subcutaneous administration of PTH stimulates bone formation and increases the BMD of long and vertebral bones [11]. PTH is thus used for osteoporosis treatments that are approved by the Food and Drug 
Administration and other organizations. Intermittent administration of PTH stimulates new bone formation in rodent cancellous bones by inhibiting osteoblast apoptosis and reducing Sost expression in osteocytes [12-14]. The results from several animal studies have indicated that intermittent PTH injection enhances osseointegration and/or increases bone volume around implants placed into long bones [15-17]. Despite this, there is only sparse scientific evidence regarding the effects of intermittent PTH administration on bone quality around implants, as the updated concept of bone quality supported by bone cells, collagen, and biological apatite is not pervasive in implant dentistry. We hypothesize that intermittent $\mathrm{PTH}$ injection enhances both bone quantity and bone quality in the bone surrounding implants. The aim of this animal study was to investigate the effects of intermittent subcutaneous administration of PTH on bone quality around implants in rat tibiae.

\section{Materials and methods}

\subsection{Animals, implant placement, and administration drugs}

Female Wistar rats were used for experimental studies (12weeks old, $\mathrm{n}=14$; Kyudo Co., Ltd, Saga, Japan). Grade IVtitanium-threaded implants that were $3.5 \mathrm{~mm}$ long and $2.0 \mathrm{~mm}$ wide and designed for rats had a thread pitch and height of $400 \mu \mathrm{m}$ and $200 \mu \mathrm{m}$, respectively [10] ( $\mathrm{n}=14$; Kyocera Co. Ltd., Kyoto, Japan). The rats were treated with systemic anesthesia $(90 \mathrm{mg} / \mathrm{kg}$ ketamine and $10 \mathrm{mg} / \mathrm{kg}$ xylazine), and an approximately $10 \mathrm{~mm}$ incision line was then prepared along the longitudinal axis of the tibiae. Skin flaps and the periosteum were elevated, and the implant was placed with its center at $7 \mathrm{~mm}$ from the end of a randomly selected side of the proximal tibiae. The skin was then sutured with 6-0 nylon (Natsume Seisakusho Co. Ltd., Tokyo, Japan). All rats were randomly divided into 2 groups 3 weeks postimplant placement, where one group intermittently received subcutaneous administrations of human PTH (pTH [1-34], BACHEM, Bubendorf, Switzerland) every 2 days ( $80 \mu \mathrm{g} / \mathrm{kg}$ [18]) (PTH; $\mathrm{n}=7$ ), and the other group received saline injections (VC; $n=7$ ) (Fig. $1 \mathrm{~A}$ and $\mathrm{B}$ ). Tibiae with associated implants were dissected at 5 weeks after the start of PTH administration. All animal experiments were performed according to the ARRIVE Guidelines (https://www. nc3rs.org.uk/arrive-guidelines).

\subsection{Microcomputed tomography (microCT)}

Dissected tibiae were fixed in 10\% neutral buffered formalin for $48 \mathrm{~h}$ at $4{ }^{\circ} \mathrm{C}(\mathrm{n}=7$ each/group). As previously described, microCT scans were conducted at $20-\mu \mathrm{m}$ voxel resolution with an energy level of $90 \mathrm{kV}$ (R_mCT2; Rigaku Co., Tokyo, Japan) [19]. The regions of interest (ROIs) were determined between $5.0 \mathrm{~mm}$ and $5.5 \mathrm{~mm}$ from the proximal tibiae along the longitudinal axis to avoid influence from the implants. To confirm PTH effects on long bone, trabecular bone volume per tissue volume (Tb.BV/TV), trabecular number (Tb.N), trabecular thickness (Tb.Th), trabecular separation (Tb.Sp), trabecular BMD (Tb.BMD), cortical bone ratio (cortical bone volume per total bone volume: $\mathrm{Cv} / \mathrm{Av}$ ), thickness of cortical bone (Ct.Th), and inner and outer perimeter of cortical bone (Ct.In.length and Ct.Ex.length, respectively) were semi-automatically measured using a TRI/3D-Bon (Ratoc System Engineering, Tokyo, Japan) according to published guidelines for microCT analyses [20].

\subsection{Histology and immunohistochemistry}

The dissected tibiae were fixed in $10 \%$ neutral buffered formalin for $48 \mathrm{~h}$ at $4{ }^{\circ} \mathrm{C}$ and then demineralized in ethylenediaminetetraacetic acid ( $\mathrm{pH}=7.3$, FUJIFILM Wako Pure Chemical Co., Osaka, Japan) at $4{ }^{\circ} \mathrm{C}$ for 28 days ( $\mathrm{n}=7$ each/group). The implants were then carefully removed with inverse rotation, paraffin-embedded, and cut into 5$\mu \mathrm{m}$-thick sections. The sections were stained for histomorphometric analyses incorporating hematoxylin and eosin (H\&E), tartrateresistant acid phosphatase (TRAP) (386 A; Sigma-Aldrich, St. Louis, $\mathrm{MO}$ ), and picrosirius red staining (Direct Red 80; Sigma-Aldrich) according to each manufacturer's protocol, and the sections were then visualized and photomicrographed under a light microscopy (Axio Scope A1; Zeiss, Oberkochen, Germany). Polarized light microscopy (Axio Lab. A1; Zeiss) was also used to detect picrosirius red-stained type I and III collagen.

Bone area and osteocytes surrounding the implants were both quantitatively evaluated by H\&E staining. Osteoclasts and collagen fibers were quantitatively assessed by TRAP and picrosirius red staining, respectively. Histomorphometric analyses were performed using Zen2 software (Zeiss) and ImageJ (version 1.49; NIH, Bethesda, MD) using photomicrographed images. In this study, the following evaluation parameters were created: 1) bone area fraction around implants: the ratio of the bone area of interest (AOI) occupied in the tissue AOI (BAF [\%]), 2) osteocyte density: the number of osteocytes in each AOI (osteocyte density [\#/ $\left.\mathrm{mm}^{2}\right]$ ), 3) osteoclast number: the number of osteoclasts per bone perimeter in each $\mathrm{AOI}(\mathrm{N} . \mathrm{Oc} / \mathrm{BS}[\# / \mathrm{mm}]), 4)$ collagen area fraction: the ratio of stained area occupied in the tissue AOI (CAF [\%]), and 5) production of type I and III collagen fibers: yellowish and greenish areas occupied in the tissue AOI (type I collagen [\%] and type III collagen [\%], respectively) [10]. AOIs were defined as the total inside and outside areas of the first, second, third and fourth threads.

Osteoblasts on bone surrounding the implants were visualized using immunostaining. Sections were deparaffinized, reacted with blocking agents, and incubated with primary antibody specific for Runt-related transcription factor 2 (Runx2) at $4{ }^{\circ} \mathrm{C}$ overnight. Rabbit anti-rat Runx2 polyclonal antibody was used as the first antibody (1:800, ab23981; Abcam, Cambridge, MA). Endogenous peroxidase activity was blocked with $3 \%$ hydrogen peroxide (FUJIFILM Wako Pure Chemical Co), and the sections were then incubated with secondary antibody for $60 \mathrm{~min}$ at room temperature, developed with 3,3-diaminobenzidine (Sigma-Aldrich), counterstained using hematoxylin, and finally mounted. Goat anti-rabbit immunoglobulin $G$ conjugated to horseradish peroxidase was used as the secondary antibody (ab6721; Abcam). The stained sections were photomicrographed using a light microscopy (Axio Scope A1; Zeiss) to quantitatively assess osteoblasts within the AOIs. The number of osteoblasts per bone perimeter in each AOI was semi-automatically counted (N.Ob/BS [\#/mm]) (Fig. 1C).

\subsection{Statistical analyses}

Normality was confirmed using the Shapiro-Wilk test. Nonparametric data were not noted. Student's $t$-test was used to compare the parametric data for the VC and PTH groups. All data are expressed as means \pm SEM. A $P$-value $<0.05$ was considered statistically significant. Systat 13.2 (Systat Software, Chicago, IL, USA) was used for all statistical analyses.

\section{Results}

\subsection{Effects of intermittent subcutaneous administration of PTH on} long bones

Intermittent PTH administration significantly increased Tb.BV/ TV $(P=0.003)$, Tb.Th $(P=0.035)$ and Tb.BMD $(P=0.018)$, and this treatment significantly decreased Tb.Sp $(P=0.004)$ compared to 


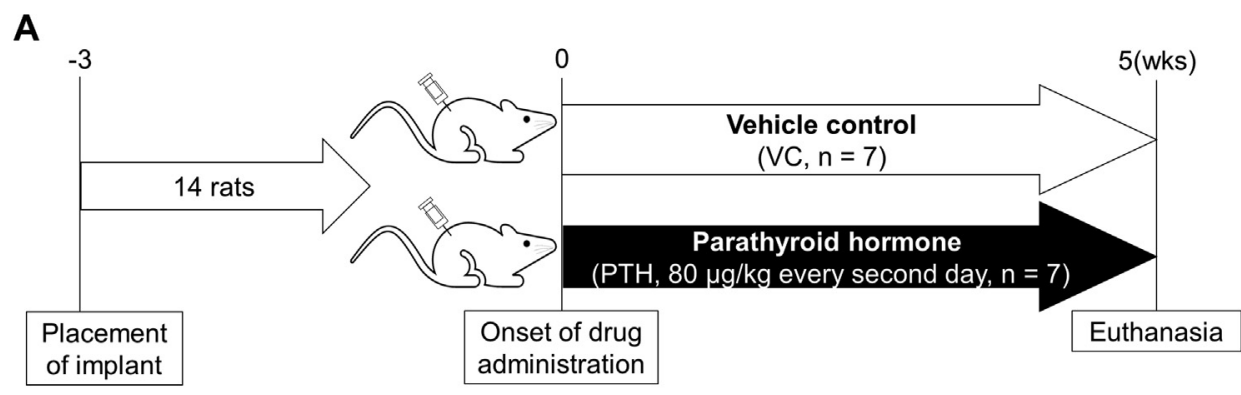

B

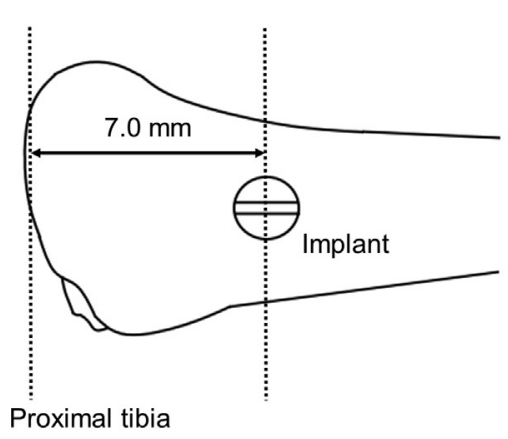

C

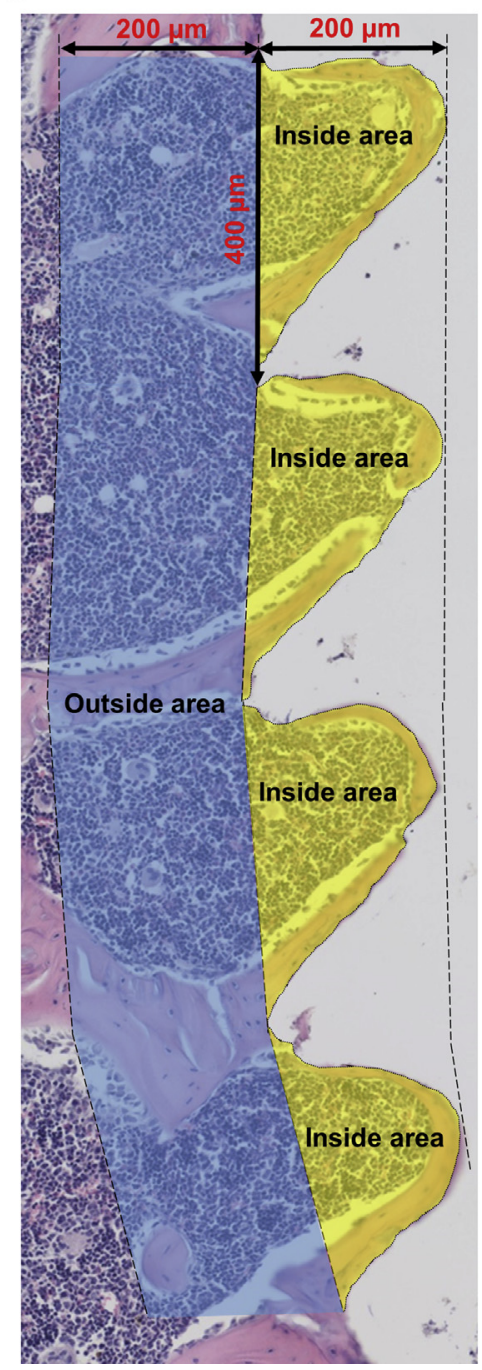

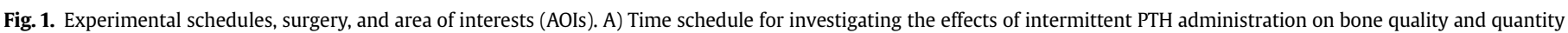

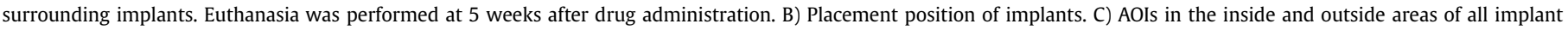
threads.

those of the VC group (Fig. 2A-F). Intermittent PTH injection significantly increased $\mathrm{Cv} / \mathrm{Av}(P=0.000)$ and Ct.Th $(P=0.009)$ (Fig. 2G and $\mathrm{H}$ ). Moreover, this treatment decreased Ct.In.length; however, the difference was not statistically significant $(P=0.081)$ (Fig. 2I). No change was observed in Ct.Ex.length between groups $(P=0.554)$ (Fig. 2J).
3.2. Effects of intermittent subcutaneous administration of PTH on bone quantity surrounding implants

Next, the effects of intermittent PTH injection on bone quantity based on bone area and the amount of collagen surrounding the implants were investigated. Intermittent PTH administration 


\section{A}

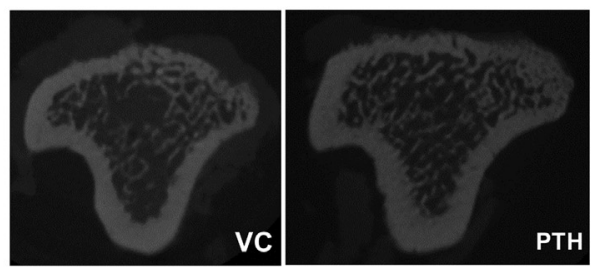

B

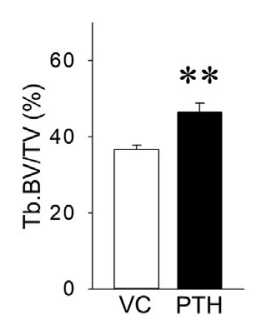

c

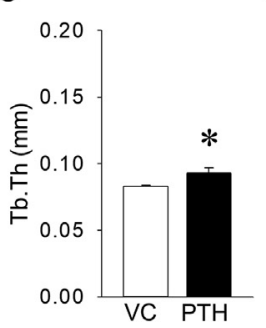

D

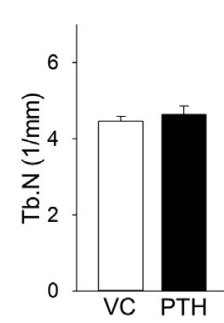

E

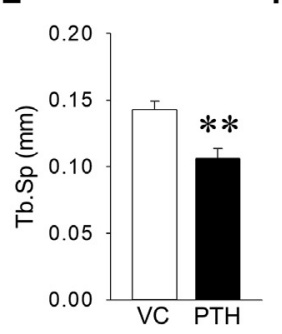

$\mathbf{F}$

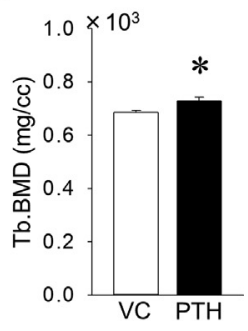

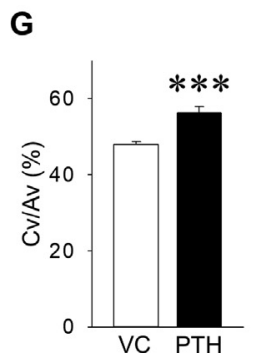

H

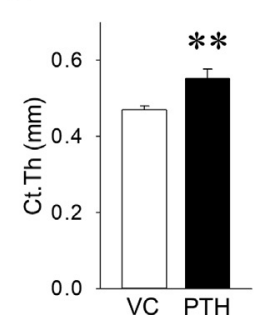

I

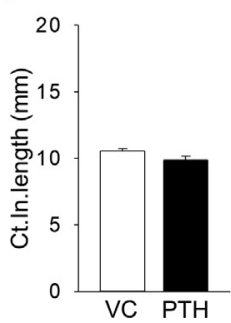

J

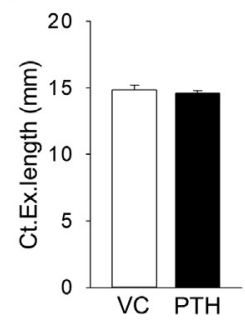

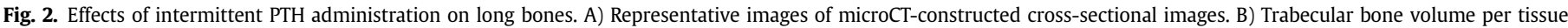

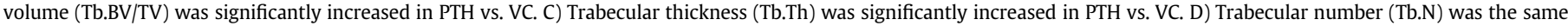

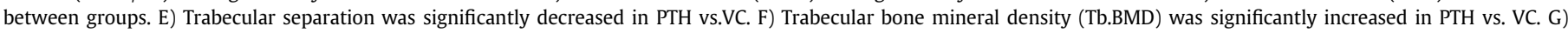

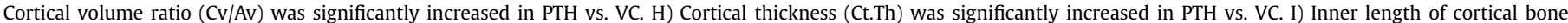

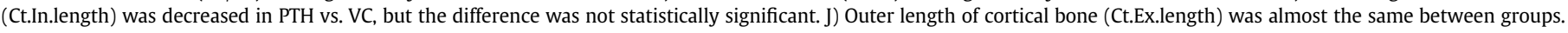
Graphs show means \pm SEM. $* P<0.05, * * P<0.01, * * * P<0.001$ (Student's $t$-test); $\mathrm{n}=7$ rats/group.

significantly increased both BAF and CAF in the total inside areas of all implant threads compared to levels in the VC group $(P=0.043$ and $P=0.023$, respectively) (Fig. $3 \mathrm{~A}-\mathrm{C}$ ); however, this treatment resulted in no significant effect on $\mathrm{BAF}$ and $\mathrm{CAF}$ in the total outside areas of all threads compared to that in the $\mathrm{VC}$ group $(P=0.995$ and $P=0.260$, respectively) (Fig. $3 \mathrm{~A}, \mathrm{D}$ and $\mathrm{E}$ ).

\subsection{Effects of intermittent subcutaneous administration of PTH on bone quality based on the types of collagen surrounding the implants}

Next, the effects of intermittent administration of PTH on bone quality based on the types of collagen surrounding the implants were investigated. Intermittent injection of PTH exerted no effect on the amount of type I collagen in the total inside area of all implant threads $(P=0.433)$; however, it did increase the amount of type III collagen compared to that in the VC group, although the difference was not statistically significant $(P=0.094)$ (Fig. 4A-C). Moreover, intermittent administration of PTH significantly increased type I collagen in the total outside area of all implant threads compared to that observed after VC administration $(P=0.002)$; however, this treatment did not affect type III collagen $(P=0.379)$ (Fig. 4A, D and E).

\subsection{Effects of intermittent subcutaneous administration of PTH on bone quality based on bone cells surrounding the implants}

Bone cells such as osteocytes, osteoclasts and osteoblasts surrounding the implants were examined. PTH administration significantly increased the number of osteocytes in both the total inside and outside areas of all implant threads compared to that in the VC group (both $P=0.000$ ) (Fig. $5 \mathrm{~A}-\mathrm{C}$ ). Counting the visualized osteoclasts on the bone surfaces revealed that PTH administration significantly increased N.Oc/BS in the total inside areas of all implant threads compared to that observed after treatment with the VC $(P=0.004)$; however, this treatment did not affect the number of osteoclasts in the total outside areas of all implant threads $(P=0.844)$ (Fig. 5D-F). Osteoblasts on the bone surface were immunostained and then counted. Intermittent administration of PTH significantly increased N.Ob/BS in both the total inside and outside areas of all implant threads compared to the values observed after VC administration $(P=0.039$ and $P=0.025$, respectively) (Fig. 5G-I).

\section{Discussion}

We demonstrated that intermittent administration of PTH for 5 weeks improved not only bone quantity, but also the updated concept of "bone quality" around implants by affecting bone cells such as osteocytes, osteoclasts, and osteoblasts and also the types of collagen fibers in rat tibiae.

In this study, implants were placed into rat tibiae rather than the jawbone. The tibial bones of large and small animals have been extensively used to evaluate bone quantity based on bone volume surrounding implants [21]. However, the use of bigger animals such as beagle dogs and rabbits is challenging for molecular-level studies such as protein and gene analyses due to the limited number of 
A
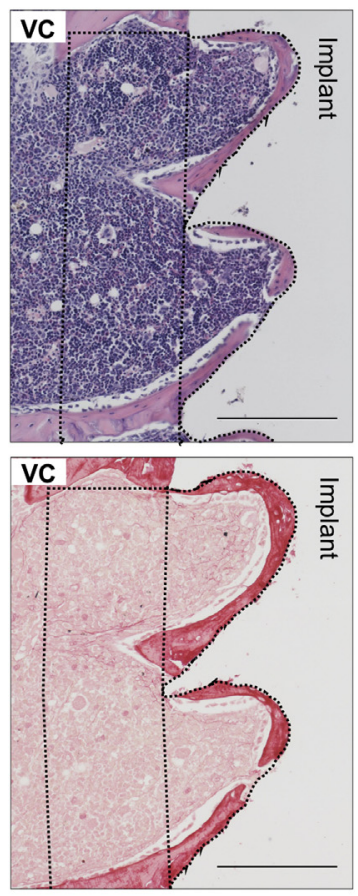

B

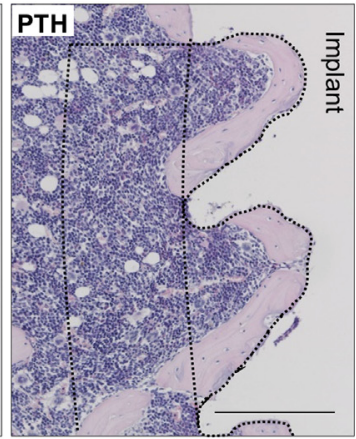

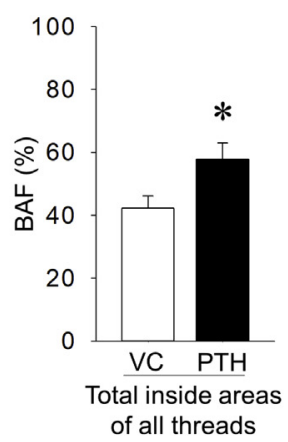

D

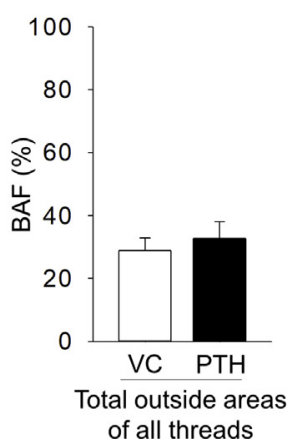

C

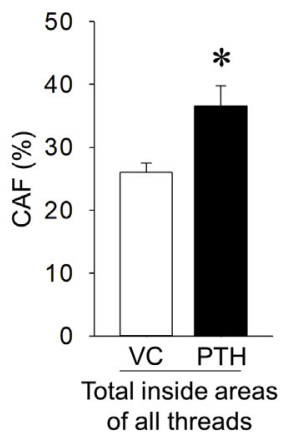

$\mathbf{E}$

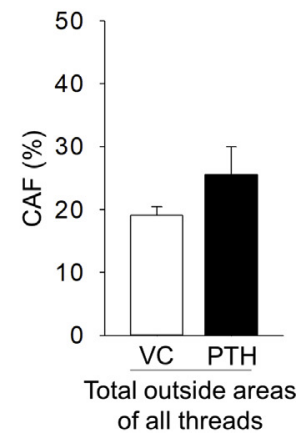

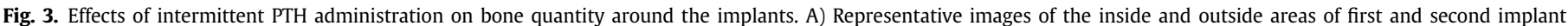

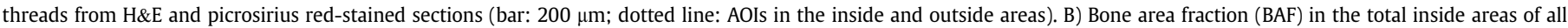

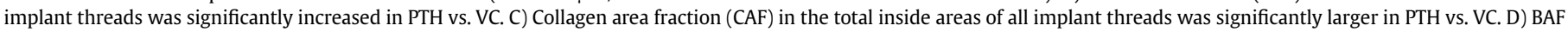

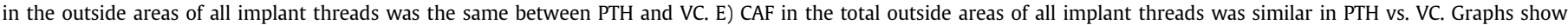
means \pm SEM. $* P<0.05$ (Student's $t$-test); $\mathrm{n}=7$ rats/group.

specific antibodies and primers for these animals, although normal diameter and length of implants can be used in studies involving larger animals. Thus, in the present study, rats were used to investigate bone quality based on bone cells and the types of collagen fibers.

Clinically, PTH is subcutaneously administered daily to treat osteoporosis. In this study, $80 \mu \mathrm{g} / \mathrm{kg}$ of human $\mathrm{PTH}(1-34)$ was subcutaneously administered to rats every second day for 5 weeks. In a previous study, subcutaneous injection of $80 \mu \mathrm{g} / \mathrm{kg}$ of human PTH(1-34) every other day significantly increased bone volume and BMD, increased Tb.Th and Tb.N, and decreased Tb.Sp in C57BL/ $6 \mathrm{~J}$ mice, although the anabolic effect was smaller than that of daily injection of the same dose [22]. Indeed, our findings in regard to structural alteration of the long bones by intermittent administration of PTH were partially supported by those of previous studies $[23,24]$, as administration duration and frequency were different compared to those in the present study ( 4 weeks and 5 times per week in mice [23], and 6 weeks and daily injection in rats [24], respectively). However, as mentioned above, the administration frequency, route, and dosage of human $\mathrm{PTH}(1-34)$ in the present study were all appropriate for evaluating the effects of PTH on bone quantity and quality surrounding implants in rat tibiae.

Intermittent injection of $40 \mu \mathrm{g} / \mathrm{kg}$ PTH for 8 weeks has been reported to enhance osseointegration and bone volume with increased Tb.Th around titanium implants placed in the tibiae of osteopenic rats that were fed a low protein diet [25]. In this previous study, interfaces between the implant and bone were excluded from the ROIs to avoid the presence of artifacts adjacent to implants caused by microCT [25]. It has been reported that detailed validation studies are requreid when metal implants are used, since they may lead to metal artifacts by microCT scan and analyses [26]. It is possible that negative effects on bone quantity around implants using microCT could be observed as a result of artifacts in the present study. Thus, histomorphometric analyses rather than microCT analyses were used to accurately evaluate bone quantity surrounding the implants. Moreover, the AOIs were separated into the inside and outside areas of all implant threads to more thoroughly investigate bone quantity and quality. Our results demonstrated that intermittent administration of PTH significantly increases bone area and also increases collagen content in the total inside areas of all implant threads while not affecting the total outside areas. Therefore, previous data partially support our results regarding bone quantity around implants, as in the previous study, the ROI represented a combination of the total inside and outside areas of the implant threads [25]. Our new findings suggest that the anabolic effect on bone quantity surrounding implants strongly depends on bone in the inside area of the implant thread rather than that in the outside area. The ratio of collagen content in bone tissue may be associated with increased bone area induced by the intermittent administration of PTH.

The concept of "bone quality" was updated 20 years ago by the $\mathrm{NIH}$ [5]; however, recognition of its importance has been impeded as BMD is believed to be equivalent to bone quality in implant treatment. Consequently, there are limited data on "bone quality" surrounding implants in implant dentistry. The present study thus focused on the updated concept of "bone quality" around implants as assessed based on the types and abundance of bone cells and collagen fibers. First, differences in the types of collagen were 
A
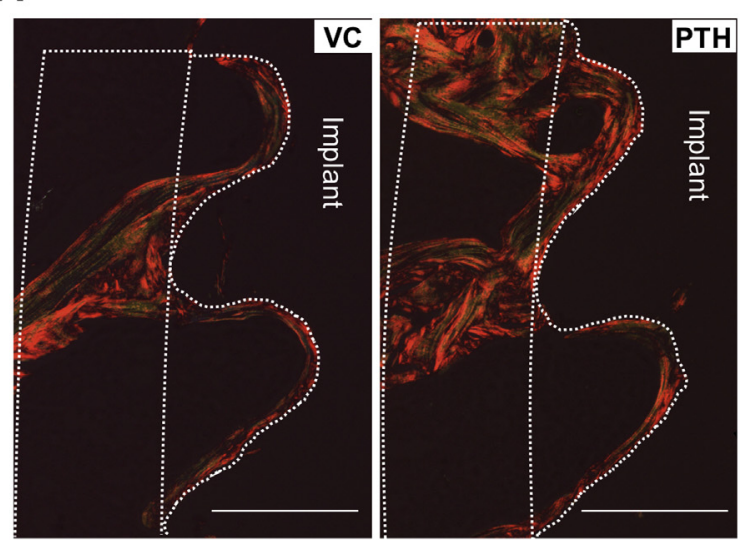

B

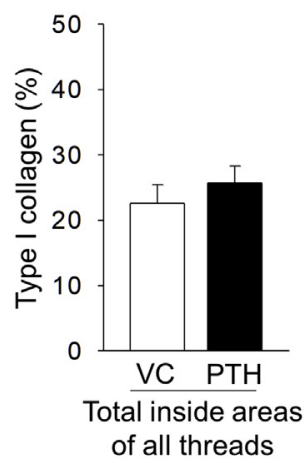

c

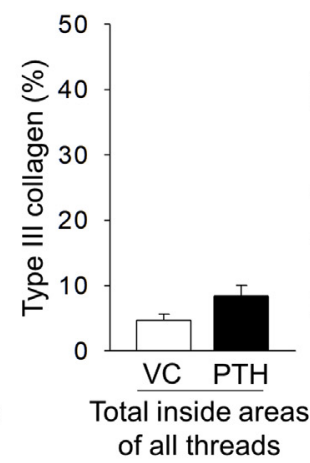

D

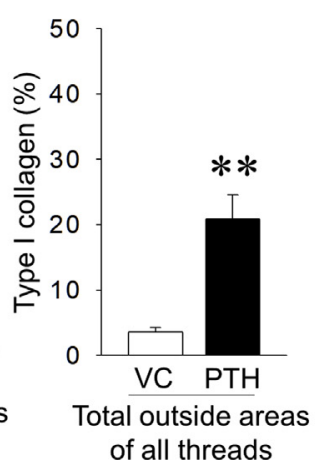

E

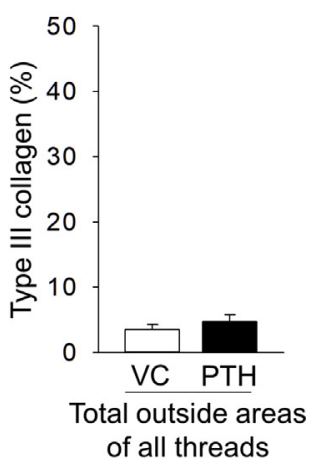

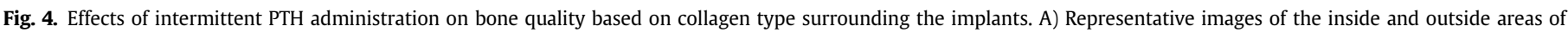

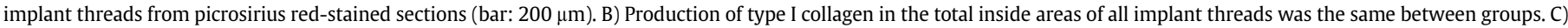

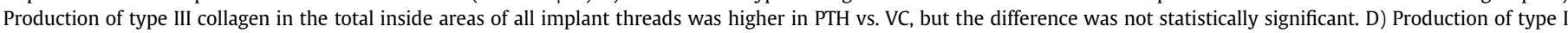

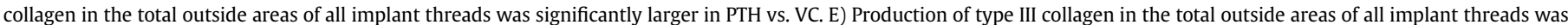
the same between groups. Graphs show means \pm SEM. ${ }^{* *} P<0.01$ (Student's $t$-test); $\mathrm{n}=7$ rats/group.

investigated using picrosirius red staining and polarized microscopy [27] due to the knowledge that collagen is a key factor controlling bone quality [28]. Interestingly, our findings show that the ratios of type I and III collagen to total collagen were different between the total inside and outside areas of all implant threads. Intermittent PTH administration has been demonstrated to active osteoblasts, resulting in the upregulation of type I collagen production [29-31]. In contrast, it has been reported that intermittent injection of human $\mathrm{PTH}(1-34)$ increased the ratio of type I collagen without altering the amount of type III collagen in bone matrix using picrosirius red staining and polarized light microcopy [32]. Therefore, our data on the induction of type I collagen production induced by intermittent PTH administration is in accordance with the previous study, although our findings differ slightly for type III collagen [32]. Type III collagen has been reported to play an important role in trabecular bone formation and maintenance by regulating osteogenesis induced by osteoblasts [33], and this suggests that both type I and III collagen are important factors for bone quality. Slight increases in type III collagen in the total inside areas of all implant threads may contribute to enhanced bone quality in this study, although it remains unknown why the ratio of type I and III collagen differed between the inside and outside areas. We speculate that these differences could be influenced by factors such as the frequency of administration, the route and dosage of PTH, the condition of the rats, and the chosen AOIs and analyzed sites (inside vs. outside areas of implant threads). Implant surface characteristics may also contribute to these differences. Further studies investigating the molecular mechanisms of collagen production induced by the intermittent administration of PTH surrounding implants are required.

Next, bone cells such as osteoblasts, osteoclasts, and osteocytes were histomorphometrically and immunohistochemically assessed to evaluate bone quality around the implants. The intermittent administration of PTH has been demonstrated to increase bone formation [34] by enhancing the differentiation of osteoblast precursors and bone lining cells into osteoblasts [35,36] and mesenchymal stem cells into osteoblasts via protein kinase $C \delta$ [37]. Intermittent injection of PTH inhibits apoptosis of both osteoblasts and osteocytes [38], possibility increasing RANKL production by osteoblasts and osteocytes and leading to increases in osteoclast numbers [39,40]. Moreover, intermittent PTH administration has been shown to negatively regulate sclerostin, a protein that inhibits bone formation [41]. Based on these modes of action of intermittent PTH administration, these previous data clearly support our present findings that intermittent administration of PTH significantly increases the number of osteoblasts and osteocytes in bone surrounding implants. An increase in the number of osteoblasts may contribute to increased bone area induced by PTH in the total inside areas of all implant threads, and an increase in the number of osteocytes may contribute to the development of an osteocyte 
A

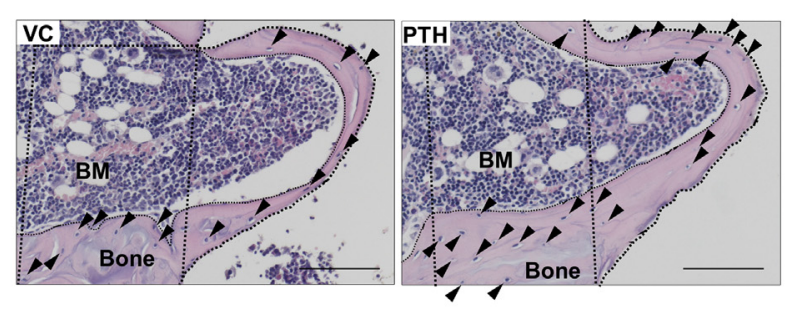

D

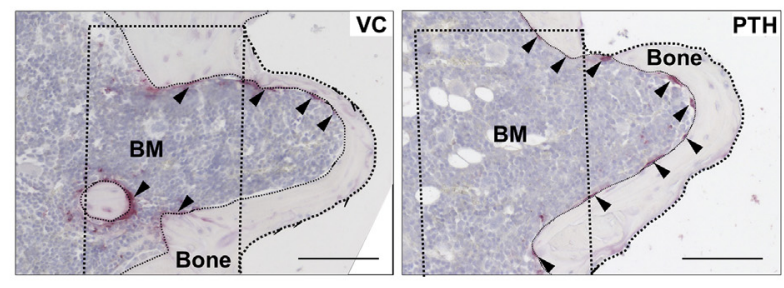

G

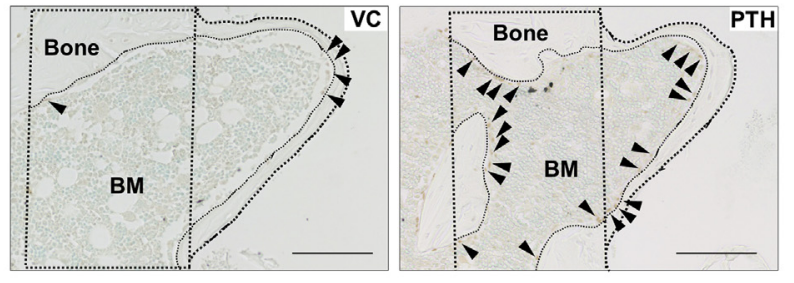

B

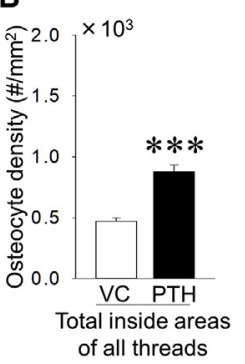

E

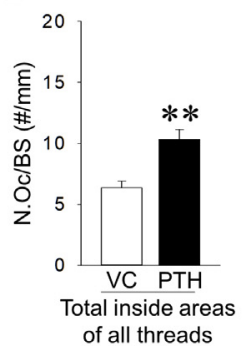

H

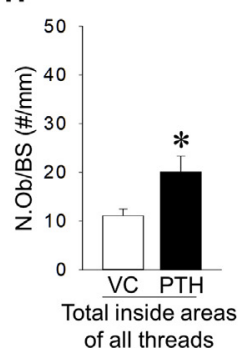

C

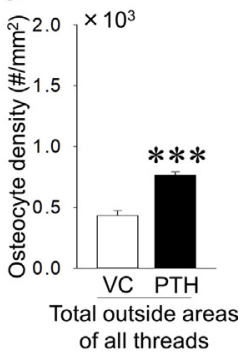

$\mathbf{F}$

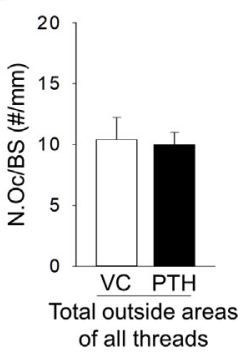

I

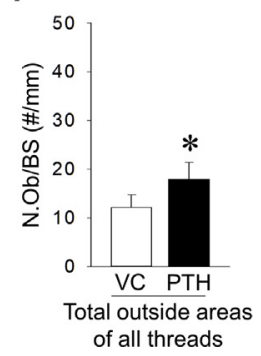

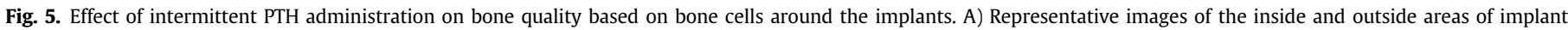

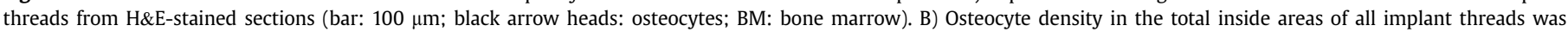

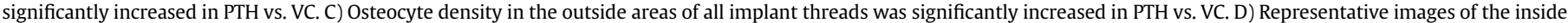

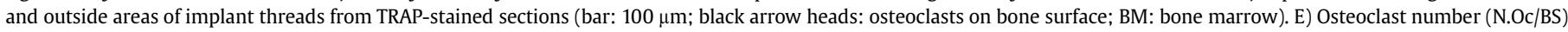

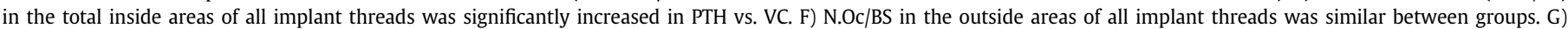

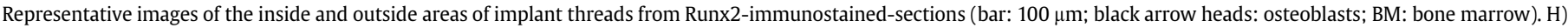

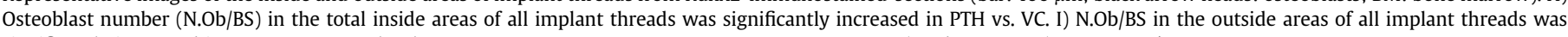
significantly increased in PTH vs. VC. Graphs show means \pm SEM. $* P<0.05$, $* * P<0.01, * * * P<0.001$ (Student's $t$-test); $\mathrm{n}=7$ rats/group.

network that is important for handling mechanical loads such as masticatory and parafunctional loads around implants. Conversely, it was recently reported that intermittent human $\mathrm{PTH}(1-34)$ administration worsened bone quality as assessed by collagen integrity in mandibular regenerative bone but not in long bones [42]. Therefore, caution is required in interpreting our results that intermittent injection of PTH improves bone quality based on collagen and bone cells. It is unclear why osteoclast number increased in the total inside but not in the outside areas of all implant threads. The production of different collagen types in the total inside and outside areas of all implant threads may be linked to the changes in osteoclast number induced by intermittent administration of PTH.

\section{Conclusions}

Within the limitations of this study (e.g., PTH administration protocol, anatomical factors, analysis methods, and methodology to evaluate bone quality), we demonstrated that subcutaneous intermittent administration of PTH significantly improved both bone quantity and bone quality based on the types and populations of bone cells and the types of collagen fibers surrounding the implants. We also demonstrated that the effects of PTH on bone quality in the total inside areas of all implant threads are larger than those in the total outside areas of all implant threads. The administration of PTH as an implant treatment may contribute to successful clinical outcomes due to improved bone quantity and quality surrounding dental implants.

\section{Ethical statement}

The animal care and experimental procedures were performed in accordance with the Guidelines for Animal Experimentation of Nagasaki University with approval from the Ethics Committee for Animal Research (Approval numbers 1608091331-2 and 1804181448-5). 


\section{Funding information}

This work was supported by grant support from JSPS KAKENHI (Grant numbers 17K19770 and 19H03844).

\section{Declaration of competing interest}

The authors declare that they have no conflicts of interest.

\section{CRediT authorship contribution statement}

Yusuke Uchida: Data curation, Formal analysis, Validation, Writing - original draft, Writing - review \& editing. Shinichiro Kuroshima: Conceptualization, Data curation, Formal analysis, Methodology, Project administration, Supervision, Validation, Writing - original draft, Writing - review \& editing. Yusuke Uto: Software, Investigation, Validation, Visualization, Writing - original draft, Writing - review \& editing. Riho Kanai: Investigation, Visualization, Writing - original draft, Writing - review \& editing. Maaya Inoue: Investigation, Visualization, Writing - original draft, Writing - review \& editing. Masayoshi Suzue: Software, Investigation, Writing - original draft, Writing - review \& editing. Takashi Sawase: Funding acquisition, Resources, Supervision, Writing original draft, Writing - review \& editing.

\section{Acknowledgements}

We thank Assistant Prof. Muneteru Sasaki, Department of Applied Prosthodontics, Graduate School of Biomedical Sciences, Nagasaki University for his excellent technical advice.

\section{References}

[1] Albrektsson T, Zarb G, Worthington P, Eriksson AR. The long-term efficacy of currently used dental implants: a review and proposed criteria of success. Int J Oral Maxillofac Implants 1986;1:11-25.

[2] Goiato MC, dos Santos DM, Santiago JF, Moreno A, Pellizzer EP. Longevity of dental implants in type IV bone: a systematic review. Int J Oral Maxillofac Surg 2014;43:1108-16.

[3] Radi IA, Ibrahim W, Iskandar SMS, AbdelNabi N. Prognosis of dental implants in patients with low bone density: a systematic review and meta-analysis. J Prosthet Dent 2018;120:668-77.

[4] Misch C. Bone density: A key determinant for clinical success. Contemporary Implant Dentistry. 2nd ed. St Louis: CV Mosby Company; 1999. p. 109-18.

[5] Osteoporosis prevention, diagnosis, and therapy. NIH Consens Statement 2000; $17: 1-45$

[6] Fyhrie DP. Summary-Measuring "bone quality". J Musculoskelet Neuronal Interact 2005:5:318-20.

[7] Kuroshima S, Nakano T, Ishimoto T, Sasaki M, Inoue M, Yasutake M, et al. Optimally oriented grooves on dental implants improve bone quality around implants under repetitive mechanical loading. Acta Biomater 2017;48: $433-44$.

[8] Kuroshima S, Yasutake M, Tsuiki K, Nakano T, Sawase T. Structural and qualitative bone remodeling around repetitive loaded implants in rabbits. Clin Implant Dent Relat Res 2015:17(Suppl 2):e699-710.

[9] Sasaki M, Kuroshima S, Aoki Y, Inaba N, Sawase T. Ultrastructural alterations of osteocyte morphology via loaded implants in rabbit tibiae. J Biomech 2015;48: 4130-41.

[10] Uto Y, Kuroshima S, Nakano T, Ishimoto T, Inaba N, Uchida Y, et al. Effects of mechanical repetitive load on bone quality around implants in rat maxillae. PloS One 2017;12:e0189893.

[11] Neer RM, Arnaud CD, Zanchetta JR, Prince R, Gaich GA, Reginster JY, et al. Effect of parathyroid hormone (1-34) on fractures and bone mineral density in postmenopausal women with osteoporosis. N Engl J Med 2001;344:1434-41.

[12] Jilka RL. Molecular and cellular mechanisms of the anabolic effect of intermittent PTH. Bone 2007:40:1434-46.

[13] Bellido T, Ali AA, Plotkin LI, Fu Q, Gubrij I, Roberson PK, et al. Proteasomal degradation of Runx2 shortens parathyroid hormone-induced anti-apoptotic signaling in osteoblasts. A putative explanation for why intermittent administration is needed for bone anabolism. J Biol Chem 2003;278:50259-72.

[14] Keller H, Kneissel M. SOST is a target gene for PTH in bone. Bone 2005;37: $148-58$.

[15] Jiang L, Zhang W, Wei L, Zhou Q Yang G, Qian N, et al. Early effects of parathyroid hormone on vascularized bone regeneration and implant osseointegration in aged rats. Biomaterials 2018;179:15-28.
[16] Almagro MI, Roman-Blas JA, Bellido M, Castañeda S, Cortez R, HerreroBeaumont G. PTH [1-34] enhances bone response around titanium implants in a rabbit model of osteoporosis. Clin Oral Implants Res 2013;24:1027-34.

[17] Daugaard H, Elmengaard B, Andreassen TT, Lamberg A, Bechtold JE, Soballe K. Systemic intermittent parathyroid hormone treatment improves osseointegration of press-fit inserted implants in cancellous bone. Acta Orthop 2012;83:411-9

[18] Kuroshima S, Entezami P, McCauley LK, Yamashita J. Early effects of parathyroid hormone on bisphosphonate/steroid-associated compromised osseous wound healing. Osteoporos Int 2014;25:1141-50.

[19] Kuroshima S, Nakajima K, Sasaki M, I. T, Sumita Y, Asahara T, et al. Systemic administration of quality- and quantity-controlled PBMNCs reduces bisphosphonate-related osteonecrosis of jaw-like lesions in mice. Stem Cel Res Ther 2019;10:209.

[20] Bouxsein ML, Boyd SK, Christiansen BA, Guldberg RE, Jepsen KJ, Müller R. Guidelines for assessment of bone microstructure in rodents using microcomputed tomography. J Bone Miner Res 2010;25:1468-86.

[21] Pearce AI, Richards RG, Milz S, Schneider E, Pearce SG. Animal models for implant biomaterial research in bone: a review. Eur Cell Mater 2007;13:1-10.

[22] Yamamoto T, Hasegawa T, Sasaki M, Hongo H, Tsuboi K, Shimizu T, et al. Frequency of teriparatide administration affects the histological pattern of bone formation in young adult male mice. Endocrinology 2016;157:2604-20.

23] Ferrari SL, Pierroz DD, Glatt V, Goddard DS, Bianchi EN, Lin FT, et al. Bone response to intermittent parathyroid hormone is altered in mice null for $\beta$ Arrestin2. Endocrinology 2005;146:1854-62.

[24] Brouwers JE, van Rietbergen B, Huiskes R, Ito K. Effects of PTH treatment on tibial bone of ovariectomized rats assessed by in vivo micro-CT. Osteoporos Int 2009;20:1823-35.

[25] Dayer R, Brennan TC, Rizzoli R, Ammann P. PTH improves titanium implant fixation more than pamidronate or renutrition in osteopenic rats chronically fed a low protein diet. Osteoporos Int 2010;21:957-67.

[26] Liu S, Broucek J, Virdi AS, Sumner DR. Limitations of using micro-computed tomography to predict bone-implant contact and mechanical fixation. J Microsc 2012;245:34-42.

[27] Junqueira LC, Bignolas G, Brentani RR. Picrosirius staining plus polarization microscopy, a specific method for collagen detection in tissue sections. Histochem J 1979;11:447-55.

[28] Kuroshima S, Kaku M, Ishimoto T, Sasaki M, Nakano T, Sawase T. A paradigm shift for bone quality in dentistry: a literature review. J. Prosthodont. Res. 2017;61:353-62.

[29] Canalis E, Centrella M, Burch W, McCarthy TL. Insulin-like growth factor mediates selective anabolic effects of parathyroid hormone in bone cultures J Clin Invest 1989;83:60-5.

[30] Onyia JE, Helvering LM, Gelbert L, Wei T, Huang S, Chen P, et al. Molecular profile of catabolic versus anabolic treatment regimens of parathyroid hormone (PTH) in rat bone: an analysis by DNA microarray. J Cell Biochem 2005;95:403-18.

[31] Chen B, Lin T, Yang X, Li Y, Xie D, Cui H. Intermittent parathyroid hormone (134) application regulates cAMP-response element binding protein activity to promote the proliferation and osteogenic differentiation of bone mesenchymal stromal cells, via the cAMP/PKA signaling pathway. Exp Ther Med 2016:11:2399-406.

[32] Pacheco-Costa R, Campos JF, Katchburian E, de Medeiros VP, Nader HB Nonaka KO, et al. Modifications in bone matrix of estrogen-deficient rats treated with intermittent PTH. Biomed Res Int 2015;2015:454162.

[33] Volk SW, Shah SR, Cohen AJ, Wang Y, Brisson BK, Vogel LK, et al. Type III collagen regulates osteoblastogenesis and the quantity of trabecular bone. Calcif Tissue Int 2014:94:621-31.

[34] Lindsay R, Zhou H, Cosman F, Nieves J, Dempster DW, Hodsman AB. Effects of a one-month treatment with $\mathrm{PTH}(1-34)$ on bone formation on cancellous endocortical, and periosteal surfaces of the human ilium. J Bone Miner Res 2007:22:495-502.

[35] Dobnig H, Turner RT. Evidence that intermittent treatment with parathyroid hormone increases bone formation in adult rats by activation of bone lining cells. Endocrinology 1995:136:3632-8.

[36] Balani DH, Ono N, Kronenberg HM. Parathyroid hormone regulates fates of murine osteoblast precursors in vivo. J Clin Invest 2017;127:3327-38.

[37] Kuo SW, Rimando MG, Liu YS, Lee OK. Intermittent administration of parathyroid hormone 1-34 enhances osteogenesis of human mesenchymal stem cells by regulating protein kinase C $\delta$. Int J Mol Sci 2017;18:2221.

[38] Jilka RL, Weinstein RS, Bellido T, Roberson P, Parfitt AM, Manolagas SC Increased bone formation by prevention of osteoblast apoptosis with parathyroid hormone. J Clin Invest 1999;104:439-46.

[39] Nakashima T, Hayashi M, Fukunaga T, Kurata K, Oh-Hora M, Feng JQ et al Evidence for osteocyte regulation of bone homeostasis through RANKL expression. Nat Med 2011:17:1231-4.

[40] Martin TJ, Sims NA. RANKL/OPG; Critical role in bone physiology. Rev Endocr Metab Disord 2015;16:131-9.

[41] Kramer I, Loots GG, Studer A, Keller H, Kneissel M. Parathyroid hormone (PTH)-induced bone gain is blunted in SOST overexpressing and deficient mice. J Bone Miner Res 2010;25:178-89.

[42] Yoshioka Y, Yamachika E, Nakanishi M, Ninomiya T, Akashi S, Kondo S, et al. Intermittent parathyroid hormone 1-34 induces oxidation and detrioration of mineral and collagen quality in newly formed mandibular bone. Sci Rep 2019;9:8041. 